\title{
Assessment of Serum Level of Vaspin in Patients with Acne Vulgaris and its Relation with Insulin Resistance
}

\author{
A.A.Ibrahim ${ }^{1}$, A.I.Mustafa ${ }^{1}$, W.A.El-halim ${ }^{2}$, R.M.Al-Razzaz ${ }^{1}$
}

${ }^{1}$ Dermatology, Venereology Dept., Faculty of Medicine, Benha Univ., Benha, Egypt

${ }^{2}$ clinical and chemical pathology Dept., Faculty of Nursing, Benha Univ., Benha, Egypt

E-Mail: Radwa.alrazzaz@gmail.com

\begin{abstract}
Skin inflammation vulgaris (AV) is a typical constant skin illness. Vaspin (instinctive fat tissue-inferred serine protease inhibitor), a novel adipocytokine. It has been recommended that vaspin has potential insulin-sharpening impacts. Vaspin assumes a part in adipoinsular hub, and might be related with insulin obstruction. This investigation expected to assess serum vaspin level in patients with skin break out vulgaris to assess its part in AV pathogenesis and its connection with insulin opposition in skin inflammation patients. This investigation was led on 52 patients experiencing skin inflammation vulgaris. Notwithstanding 32 as control gathering. Patients were selected from the outpatient facility of Dermatology and Andrology Department of Benha University Hospitals. All contemplated people were exposed to finish history taking, dermatological assessment and research facility examinations included: assessment of serum levels of vaspin, fasting blood glucose and Homeostasis Model Assessment of Insulin Resistance (HOMA-IR). Blood glucose, Fasting insulin and HOMA-IR were altogether higher in AV situations when contrasted with control gatherings. AV bunch demonstrated fundamentally lower vaspin when contrasted with control gathering. HOMA-IR indicated huge positive relationship with grades of AV patients. Vaspin demonstrated huge negative connection with HOMA-IR in AV gathering. There is a function of serum vaspin in the pathogenesis of skin break out vulgaris. AV bunch demonstrated fundamentally lower vaspin when contrasted with control gathering. HOMA-IR was altogether higher in AV situations when contrasted with control gatherings. We found a measurably critical relationship between HOMA-IR and grades of AV patients. Vaspin demonstrated huge negative relationship with HOMA-IR in AV gathering.
\end{abstract}

Keywords: Vaspin, Acne vulgaris, Insulin resistance.

\section{Introduction}

Skin break out vulgaris (AV) is the most wellknown skin issue found in wandering dermatology rehearses paying little heed to sex, skin tone or nationality [1].

$\mathrm{AV}$ is a typical, ongoing, fiery, facial skin problem that can influence people from any race, identity, or social foundation, the beginning of AV is generally quickly previously or during early immaturity; notwithstanding, a few cases start in last adolescence [2].

Skin inflammation is assessed to influence $9.4 \%$ of the worldwide populace, making it the eighth most pervasive sickness around the world. Epidemiological examinations have shown that skin inflammation is generally normal in postpubescent adolescents, with young men most habitually influenced, especially with more serious types of the illness [3].

Skin inflammation is a multifactorial infection including the pilosebaceous units. Changed follicular keratinization, colonization of pilosebaceous units by Propionibacterium acnes, expanded sebum creation, hormonal impacts and irritation have been proposed in pathogenesis of skin inflammation vulgaris. IGF-1 has been proposed to be one of the middle people of skin inflammation vulgaris. Skin break out as a piece of disorder like polycystic ovary (PCOS) and hyperandrogenism insulin obstruction acanthosis nigricans (HAIR-AN) demonstrate insulin opposition in skin inflammation [4].

Fat tissue secretes adipokines with proinflammatory movement, for example, leptin, visfatin and resistin, or calming properties, for example, adiponectin and vaspin, which work as flagging organizations that discuss fat tissue with various organs (mind, liver, lymphoid, and so on) and direct the digestion. In states of stoutness, its discharge adds to a low yet consistent condition of irritation, which advances atherosclerosis and IR [5].

VASPIN is a Visceral Adipose Tissue Derived Serine Protease Inhibitor, one of the more as of late found adipokine. VASPIN has insulin detecting, against atherogenic and calming exercises by hindering the Nuclear Factor-kappa B (NF-kB) in this manner restraining the creation of cytokines and attachment particles [6].

The investigation intended to assess serum vaspin level notwithstanding fasting insulin and fasting blood glucose levels in patients with skin inflammation vulgaris and appraisal of its clinical centrality and connection to IR.

\section{Patients and methods Type of the Study}

A case- control study.

\section{Study Population}

This study that was conducted on 52 patients suffering from AV (Group A) and 32 apparently healthy individuals of matched age and sex as a control group (Group B). Patients were recruited from the outpatient clinic of Dermatology and Andrology Department of Benha University Hospitals between [June 2019]. 


\section{Administrative Design and Ethical Considerations}

The study was approved by the local ethic committee of Benha Faculty of Medicine. Informed consent was obtained from each individual before sample collection.

\section{Inclusion Criteria}

Patients with different degrees of severity of AV according to Global Acne Grading system (GAGS) [7].

\section{Exclusion Criteria}

1- Patients with AV on systemic therapy that affect blood glucose level eg: systemic retinoids and hormonal therapy.

2- Patients with positive history of eating disorders eg: Bulimia nervosa and anorexia nervosa.

3- Patients with acute or chronic infection.

4- Patients with diabetes mellitus (DM).

5- Patients with hypertension or ischemic heart disease.

6- Patients with thyroid disorders, chronic renal or liver diseases.

7- Patients younger than 18 years of age.

8- Female patients with menstrual irregularities.

9- Pregnant and breastfeeding females.

\section{Methods}

All patients were subjected to the followings Full history taking

- Personal history: Name, age, sex, occupation, residence, special habits of medical importance, marital status.

\section{Clinical examination}

- Patients were graded according to GAGS into three groups:

Mild (18 patients), moderate (18 patients) and severe (16 patients).

The total severity score was derived from the summation of six regional subscores. Each was derived by multiplying the factor for each region; (factor for forehead and each cheek was 2, chin and nose was 1 and chest and upper back was 3); by the most heavily weighted lesion within each region (1 for comedones, 2 for papules, 3 for pustules and 4 for nodules). A score of (1-18) is considered mild; (1930) moderate; (31-38) severe; and $>39$ very severe [7].

\section{Laboratory investigations}

All studied subjects were tested for:

1- Serum level of vaspin.

2- Serum level of fasting insulin level.

3- Fasting blood glucose level.

\section{Sampling}

Five ml venous blood was collected from each subject by clean venipuncture using disposable plastic syringe and placed on plain tube (without anticoagulant) for serum separation. The tube was left at room temperature for 30 minutes till coagulation, and then was centrifuged (at $1500 \mathrm{rpm}$ for 15 minutes).

The resultant serum was stored at $-20^{\circ} \mathrm{C}$ for further testing.

\section{Vaspin}

A double-antibody sandwich ELISA (Enzyme Linked Immune Sorbent Assay) was used to detect serum level of vaspin using a commercial Human vaspin ELISA Kit for research use only (Cat \#: 20112-0922, SunRedBio, China).

\section{Fasting Insulin}

A double-antibody sandwich ELISA was used to detect serum level of insulin using a commercial Human Insulin Quantitative ELISA Kit for diagnostic use (Cat \#: 10801, IMMUNOSPEC, USA).

\section{Fasting blood glucose level}

A commercial diagnostic standard colorimetric method was used to estimate serum blood glucose level.

\section{Insulin resistance}

Insulin resistance was calculated according to the Homeostasis Model Assessment of Insulin Resistance (HOMA-IR) formula: [Fasting glucose $(\mathrm{mmol} / \mathrm{L}) \mathrm{x}$ Fasting serum insulin $(\mathrm{mIU} / \mathrm{mL})] / 22.5$.

\section{Statistical Analysis}

The collected data were revised, coded, tabulated and introduced to a PC using Statistical package for Social Science (IBM Corp. Released 2017. IBM SPSS Statistics for Windows, Version 25.0. Armonk, NY: IBM Corp.). Data were presented and suitable analysis was done according to the type of data obtained for each parameter.

\section{Descriptive statistics}

1- Mean, Standard deviation ( \pm SD) for numerical data.

2- Frequency and percentage of non-numerical data.

3- Shapiro test was done to test the normality of data distribution.

\section{Analytical statistics}

- Student T Test was used to assess the statistical significance of the difference between two study group means.

- For the comparison of the three groups' means, one way analysis of variance (ANOVA) was used.

- Chi-Square test was used to examine the relationship between two qualitative variables

- Fisher's exact test: was used to examine the relationship between two qualitative variables when the expected count is less than 5 in more than $20 \%$ of cells.

- Correlation analysis: To assess the strength of association between two quantitative variables. 
The correlation coefficient defines the strength and direction of the linear relationship between two variables.

- N.B: $p$ is significant if $<0.05$ at confidence interval $95 \%$.

\section{Results}

The AV group mean age was 20.1 years, they were 25 females $(48.1 \%)$ and 27 males (51.9\%). In addition to 32 healthy control group, their mean age was 20.7 years, they were 16 males $(50 \%)$ and 16 females $(50 \%)$.Studied patients and control groups were age and sex matched ( $p$ value $=0.381,0.864$ respectively).Mean BMI of AV group was 27.8 $\mathrm{kg} / \mathrm{m} 2$, while that of control group was $23.7 \mathrm{~kg} / \mathrm{m} 2$, with significant difference between both groups $(\mathrm{p}=0.002)$ shown in Table (1).

AV patients were graded into three groups; mild, moderate and severe according to GAGS. Mild cases represented $18(34.6 \%)$, moderate cases represented $18(34.6 \%)$ while severe cases were observed only in (16) $30.8 \%$ of all studied patients Fig (1).

Fasting insulin, blood glucose and HOMA-IR were significantly higher in $\mathrm{AV}$ patients when compared to control groups $(\mathrm{p}=0.006,0.001,0.005$ respectively) as shown at Table (2).

Acne vulgaris group showed significantly lower serum vaspin level when compared to control group $(\mathrm{p}<0.001)$ as shown in Table (3).

Receiver operating characteristic (ROC) curve of HOMA-IR and serum vaspin level was conducted for discrimination between $\mathrm{AV}$ patients and control groups. HOMA-IR showed good AUC (AUC $=0.836$ ). At cut off value of 0.75 , sensitivity was $92.3 \%$, specificity was $68.8 \%$, PPV was $82.8 \%$, NPV was $84.6 \%$, and accuracy was $83.3 \%$. Serum Vaspin level showed excellent AUC (AUC $=0.977$ ). At cut off value of 120.8 , sensitivity was $90.4 \%$, specificity was $87.5 \%$, PPV was $92.2 \%$, NPV was $84.9 \%$, and accuracy was $89.3 \%$ as shown in Table (4) and Fig (2).

HOMA-IR increased significantly with increased acne severity ( $\mathrm{p}$ value $<0.001$ ) Table (5).

Vaspin level decreased significantly with increased acne severity (p value<0.001) Table (6).

Serum Vaspin level showed significant negative correlation with HOMA-IR in AV group as shown in Table (7).

Table (1) Socio- demographic and anthropometric data between cases and control groups.

\begin{tabular}{llccccc}
\hline variable & & \multicolumn{2}{c}{ Control } & \multicolumn{2}{c}{ Patients } & P \\
& & \multicolumn{2}{c}{$\mathbf{N = 3 2}$} & \multicolumn{2}{c}{ N2 } & \\
\hline Age $($ years $)$ & mean \pm SD & 20.7 & 3.1 & 20.1 & 2.6 & 0.381 \\
Males & $\mathrm{N}, \%$ & 16 & $50 \%$ & 25 & $48.1 \%$ & 0.864 \\
Females & $\mathrm{N}, \%$ & 16 & $50 \%$ & 27 & $51.9 \%$ & \\
BMI $\left(\mathbf{k g} / \mathbf{m}^{\mathbf{2}}\right)$ & mean \pm SD & 23.7 & 7.1 & 27.8 & 3.8 & 0.002 \\
\hline
\end{tabular}

$\mathrm{SD}$, standard deviation; student $\mathrm{t}$ test was used for numerical parameters; Chi square test was used for categorical parameters.

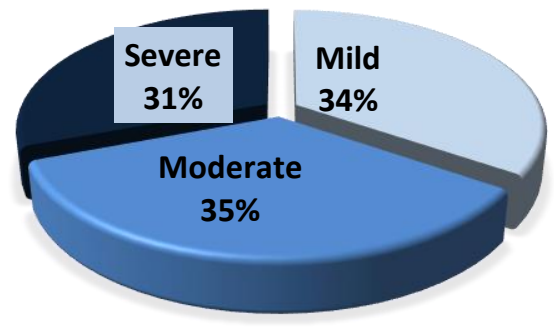

Fig (1) Grades of acne severity in all studied patients.

Table (2) Comparison of laboratory data (fasting insulin, fasting blood glucose, HOMA-IR) between cases and control groups.

\begin{tabular}{|c|c|c|c|c|c|}
\hline \multirow[t]{2}{*}{ laboratory data } & \multicolumn{2}{|c|}{$\begin{array}{c}\text { Control } \\
\mathrm{N}=32\end{array}$} & \multicolumn{2}{|c|}{$\begin{array}{c}\text { patients } \\
\mathrm{N}=52\end{array}$} & \multirow[t]{2}{*}{$\mathbf{P}$} \\
\hline & mean & \pm SD & Mean & \pm SD & \\
\hline Fasting Insulin (uIU/ml) & 4.5 & 1.1 & 8.2 & 2.2 & 0.006 \\
\hline Fasting Blood Glucose (mg/dl) & 106 & 12 & 119.5 & 16.7 & 0.001 \\
\hline HOMA-IR & 0.9 & 0.3 & 2.7 & 0.8 & 0.005 \\
\hline
\end{tabular}

$\mathrm{SD}$, standard deviation; student $\mathrm{t}$ test was used for numerical parameters. 
Table (3) Comparison of serum Vaspin level between all studied groups.

\begin{tabular}{lcccccc}
\hline variable & \multicolumn{2}{c}{$\begin{array}{c}\text { Control } \\
\mathbf{N = 4 0}\end{array}$} & \multicolumn{2}{c}{ patients } & p \\
& & \multicolumn{2}{c}{$\mathbf{4 0}$} & p \\
\hline Vaspin & mean \pm SD & 478.2 & 117.7 & 93.8 & 25.7 & $<0.001$ \\
$(\mathbf{p g} / \mathbf{m l})$ & Range & 110.7 & 670.2 & 59.8 & 187.9 & \\
\hline
\end{tabular}

$\mathrm{SD}$, standard deviation; student $\mathrm{t}$ test was used for numerical parameters.

Table (4) AUCs and performance features of HOMA-IR and serum vaspin levels for discrimination between AV patients and control groups.

\begin{tabular}{lll}
\hline Variable & HOMA-IR & Vaspin \\
\hline AUC & 0.836 & 0.977 \\
Cut off & 0.75 & 120.8 \\
Sensitivity (\%) & 92.3 & 90.4 \\
Specificity (\%) & 68.8 & 87.5 \\
PPV (\%) & 82.8 & 92.2 \\
NPV (\%) & 84.6 & 84.9 \\
Accuracy (\%) & 83.3 & 89.3 \\
\hline
\end{tabular}

AUC, area under ROC, OC, receiver operating curve; PPV, positive predictive value; NPV, negative predictive value.

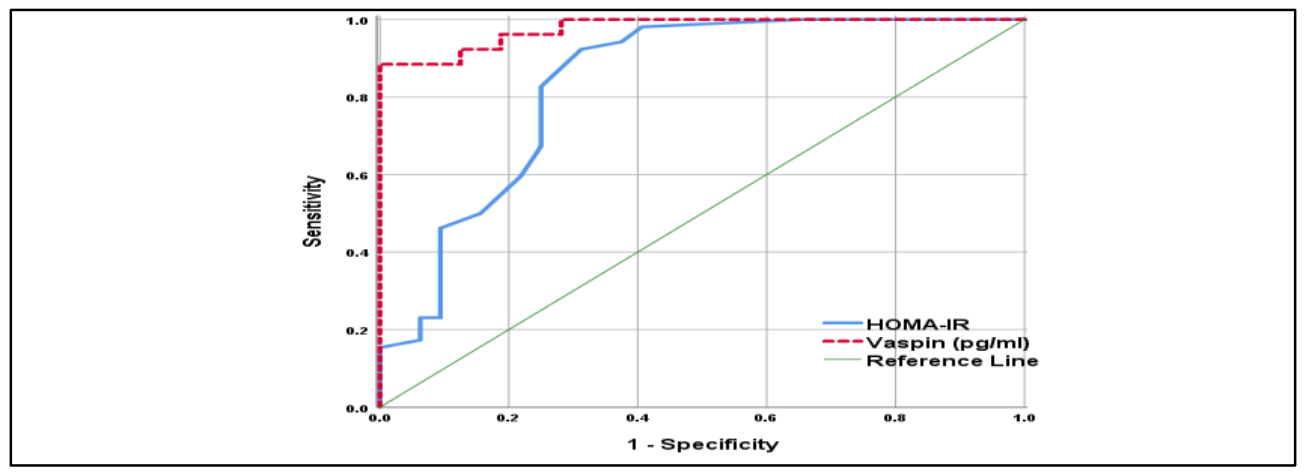

Fig (2) ROC curve of HOMA-IR and serum vaspin level for discrimination between AV patients and control groups.

Table (5) Comparison of HOMA-IR according to Grading of AV group.

\begin{tabular}{lcccccc}
\hline Variable & \multicolumn{4}{c}{ HOMA-IR } & \multicolumn{2}{c}{ p } \\
& & N & Mean & \pm & SD & \\
\hline Grading & Mild & 18 & 0.9 & \pm & 0.3 & $<0.001$ \\
& Moderate & 18 & 1.3 & \pm & 0.4 & \\
& Severe & 16 & 6.2 & \pm & 2.0 & \\
\hline
\end{tabular}

$\mathrm{SD}$, standard deviation; student $\mathrm{t}$ test was used for numerical parameters.

Table (6). Comparison of serum vaspin level according to Grading of AV group.

\begin{tabular}{lccccccc}
\hline Variable & & \multicolumn{3}{c}{ Vaspin concentration $(\mathbf{p g} / \mathbf{m L})$} & \multirow{2}{*}{$\mathbf{P}$} \\
\cline { 3 - 6 } & & $\mathbf{N}$ & mean & $\mathbf{5}$ & $\mathbf{S D}$ & & \\
\hline Grading & Mild & 18 & 118.6 & $\mathbf{\pm}$ & 27.1 & $<0.001$ \\
& Moderate & 18 & 89 & \pm & 7.5 & \\
& Severe & 16 & 71.4 & $\mathbf{\pm}$ & 6.7 & \\
\hline
\end{tabular}

$\mathrm{SD}$, standard deviation; student $\mathrm{t}$ test was used for numerical parameters. 
Table (7) Correlations of HOMA-IR with serum vaspin level in AV group.

\begin{tabular}{lll}
\hline variable & Vaspin & \\
\cline { 2 - 3 } & $\mathbf{r}$ & $\mathbf{P}$ \\
\hline HOMA-IR & -0.828 & $<\mathbf{0 . 0 0 1}$ \\
\hline
\end{tabular}

\section{r, Pearson's correlation coefficient.}

\section{Discussion}

Aftereffects of the current investigation demonstrated that, mean period of AV bunch was 20.1 years. These information agree with the discoveries that $\mathrm{AV}$ is a typical sickness happening all through the pre-adulthood and the grown-up a very long time as in this period the two young men and young ladies go through hormonal changes. Specifically, androgens increment in both genders and cause sebaceous organs in the skin to grow and deliver more sebum. The expanded sebum makes the follicles become obstructed, prompting skin break out sores [8].

Aftereffects of the current examination demonstrated female prevalence (51.9\%) among patients.

Females power might be ascribed to hereditary and hormonal variables impacts. Such perception was correspondingly detailed in another investigation in Ismailia city, Egypt, where skin break out predominance was more in females $(56 \%)$ than guys (44\%) [9].

Consequences of the current examination appeared, mean BMI of AV bunch was factually fundamentally higher than that of control group.These results concurred with Matilainen et al. [10] who indicated a measurably huge distinction among cases and control patients with respect to BMI.

Additionally Del Prete et al. [11] furnished proof that Italian patients influenced with skin inflammation had a high BMI.

In the current investigation Grades were evaluated for all contemplated AV cases, $34.6 \%$ were mellow, $34.6 \%$ were moderate and $30.8 \%$ were serious. This is like the consequences of studies by El-Tonsy et al. [12] who detailed that with respect to the seriousness of the sickness, the infection was gentle in $46 \%$, moderate in $32 \%$, and extreme in $22 \%$ of examined patients.

Consequences of the current investigation indicated that, blood glucose was essentially higher in AV situations when contrasted with control gatherings. These outcomes concurred with the aftereffects of the examination led by Nagpal et al. [13]; they discovered expanded blood glucose levels in AV patients contrasted with controls.

Consequences of the current examination demonstrated that fasting insulin levels were altogether higher in AV situations when contrasted with control bunches which concurred with aftereffects of the investigation directed by Emiroğlu et al. (14); their outcomes indicated a factually higher fasting insulin level in AV patients; particularly patients with extreme AV than controls.

In repudiate additionally with an examination done by Balta et al. [15] on $35 \mathrm{AV}$ cases and controls. They didn't locate any critical contrast in insulin levels among cases and controls.

Consequences of the current examination indicated that, HOMA-IR was essentially higher in AV situations when contrasted with control gatherings $(p=0.005)$. This is like the consequences of studies by Nagpal et al. [13] who indicated altogether high estimations of HOMA-IR in cases with skin break out contrasted with controls.

Additionally, an examination led by Emiroğlu et al. [14] in 243 skin inflammation patients, it was discovered that the HOMA-IR esteem was fundamentally higher than that in 156 control patients $(\mathrm{p}<0.001)$.

In the current examination, $\mathrm{AV}$ bunch indicated fundamentally lower vaspin when contrasted with control gathering (mean $=93.8$ versus 478.2 , $\mathrm{p}<0.001$ ). Which might be clarified by presence of IR in AV patients which is contrarily connected with serum vaspin level. Serum vaspin level was higher in controls because of its calming job that guard against aggravations [16].

As far as we could possibly know, no distributed examinations were found to think about the outcomes between AV patients concerning serum vaspin levels.

In our investigation HOMA-IR indicated critical positive connection with grades of $\mathrm{AV}$ patients. This comes as per Abdelmawla et al. [17] who found a factually huge positive relationship among IR and $\mathrm{AV}$ and a relationship between skin break out seriousness and HOMA levels.

In our investigation, serum Vaspin level indicated critical negative connection with HOMAIR in AV groupThis comes as per past information that demonstrated relationship of vaspin with HOMA-IR [18].

Additionally, Jian et al. [19] indicated that the serum VASPIN fixation was essentially connected with homeostasis model appraisal of insulin obstruction.

Also, Sathyaseelan et al. [20] credited the expansion in VASPIN might be a compensatory reaction to threaten the activity of other obscure proteases that are up-managed in stoutness and in conditions of insulin obstruction; consequently, this 
up-guideline might be a guarded component against insulin opposition.

However, Suleymanoglu et al. [21] detailed Vaspin levels were decidedly related with insulin resistantace.

On other hand, Aust et al. [22] neglected to show a straightforward relationship between's serum vaspin levels and IR.

\section{Conclusion}

There is a function of serum vaspin in the pathogenesis of skin break out vulgaris. AV bunch demonstrated altogether lower vaspin when contrasted with control gathering. HOMA-IR was essentially higher in AV situations when contrasted with control gatherings. We found a measurably critical relationship between HOMA-IR and grades of AV patients. Vaspin demonstrated huge negative connection with HOMA-IR in AV gathering.

\section{References}

[1] L.F. Eichenfield, J.R. Del, A.J. Mancini Evolving perspectives on the etiology and pathogenesis of acne vulgaris. Journal of drugs in dermatology: JDD, Vol.14(3), PP.263$272,2015$.

[2] J.Q. Del Rosso, The role of skin care as an integral component in the management of acne vulgaris: part 1: the importance of cleanser and moisturizer ingredients, design, and product selection. The Journal of clinical and aesthetic dermatology, Vol.6(12), PP. 19,2013.

[3] J.K. Tan ,K. Bhate A global perspective on the epidemiology of acne. Br J Dermatol ,Vol.172 (1), PP. 3-12,2015.

[4] W. Chen, O.B. Pietsch, J.B. Hong ,Acne associated syndromes: models for better understanding of acne pathogenesis. J Eur Acad Dermatol Venereol, Vol.25 (6), PP. 637646,2011.

[5] L. Fontana, J.C. Eagon, M.E. Trujillo , Visceral fat adipokine secretion is associated with systemic inflammation in obese humans. Diabetes, Vol.56,PP.1010- 1013,2007.

[6] M.A Kobat, A. Celik, M. Balin , he investigation of serum vaspin level in atherosclerotic coronary artery disease. J Clin Med Res, Vol.4(2), PP.110-13,2012.

[7] A. Doshi, A. Zaheer, M.J. Stiller A comparison of current acne grading systems and proposal of a novel system. Int J Dermatol, Vol.36 (6), PP. 416- 418, 1997.

[8] Y.L. Kong , H.L. Tey, Treatment of acne vulgaris during pregnancy and lactation. Drugs, Vol.73(8), PP.779- 787,2013.

[9] A. Shedid, Epidemiologic study of acne valgaris among secondary schools in Ismailia governorate. Egypt. Thesis, Faculty of Medicine Ain Shams University,Vol.25(8),PP.213225,2011.

[10] V. Matilainen, P. Koskela, S.KeinanenKiukaanniemi, Early androgenetic alopecia as a marker of insulin resistance. Lancet; ,Vol.356,PP.1165-1166,2000.

[11] M. Del Prete, M.C. Mauriello, C. Faggiano Insulin resistance and acne: a new risk factor for men? Endocrine,Vol.42, PP.555-560,2012.

[12] T. El-Tonsy, M. Mohammed, Y.Hamed,). Bacteriological study of Acne Vulgaris in Cairo Egypt. The Egyptian Journal of Hospital Medicine, Vol.72(9),PP. 5203-5209,2018.

[13] M. Nagpal, D. De , S. Handa, Insulin resistance and metabolic syndrome in young men with acne. JAMA Dermatol,Vol.152(4), PP. 399404,2016.

[14] N Emiroğlu, F.P. Cengiz, F.Kemeriz Insulin resistance in severe acne vulgaris. Postepy Dermatol Alergol,Vol.32(4), PP. 281285,2015 .

[15] I. Balta, O. Ekiz, P. Ozuguz, Insulin resistance in patients with post-adolescent acne. Int $\mathbf{J}$ Dermatol,Vol.54, PP.662-6,2015.

[16] J.T. Heiker Vaspin (serpinA12) in obesity, insulin resistance, and inflammation. J Pept Sci, Vol.20(5), PP.299-306,2014.

[17] M.Y. Abdelmawla, A.M. Esawy, E. Khater, Insulin resistance in androgenetic alopecia and acne vulgaris. Egypt J Dermatol Venerol,Vol.39, PP.83-8,2019

[18] H.M. Chang, H.J. Lee, H.S. Park, Effects of Weight Reduction on Serum Vaspin Concentrations in Obese Subjects: Modification by Insulin Resistance. Obesity,Vol.18(11),PP. $2105-2110,2016$.

[19] W. Jian, W. Peng, S. Xiao, Role of serum vaspin in progression of type 2 diabetes: A 2Year Cohort Study. PLOS ONE, Vol.9(4), PP. e94763,2010.

[20] A.J.Sathyaseelan,P.S.Adole,M.Wyawahar Assessment of Serum VASPIN Levels among Type 2 Diabetes Mellitus Patients with or without Acute Coronary Syndrome. Journal of clinical and diagnostic research: JCDR, Vol.10(12), PP.BC07-BC10,2014.

[21] S. Suleymanoglu, E .Tascilar, O. Pirgon, Vaspin and its correlation with insulin sensitivity indices in obese children. Diabetes Res Clin Pract ,Vol.84,PP. 325-328,2009.

[22] Aust G, Richter O, Rohm S, Kerner C, Hauss J, et al. (2009) Vaspin serum concentrations in patients with carotid stenosis. Atherosclerosis 204: 262-266. 